\title{
EXACT SOLUTIONS OF THE KLEIN-GORDON EQUATION FOR THE Q-DEFORMED MORSE POTENTIAL USING NIKIFOROV-UVAROV METHOD
}

\author{
Said Laachir and Aziz Laaribi \\ L'Académie régionale de l'éducation et de la formation de la région du Grand \\ Casablanca, \\ Lycée qualifiant Zineb Ennefzaouia- Casablanca \\ Morocco
}

\begin{abstract}
In this work, we solve the Klein-Gordon $(K G)$ equation for the general deformed Morse potential with equal scalar and vector potentials by using the Nikiforov-Uvarov (NU) method, which is based on the solutions of general second-order linear differential equation with special functions. The energy eigenvalues and corresponding normalized eigenfunctions are obtained. It is found that the eigenfunctions can be expressed by the Laguerre polynomials. Our solutions have a good agreement with earlier study.
\end{abstract}

\section{KEYWORDS}

Klein-Gordon equation, Nikiforov-Uvarov method, exact solutions, energy eigenvalues, eigenfunctions

\section{INTRODUCTION}

The Klein-Gordon equation describes relativistic spin-zero particles, plays an important role in mathematical physics. The equation has attracted much attention in studying solitons [1] in condensed matter physics, in investigating the interaction of solitons in a collisionless plasma. Many different techniques such as the supersymetric approach (SUSY) [2], the Variational method [3], the Functional Analysis method [4] and the Asymptotic Iteration method [5-8] have been introduced to solve the KG equation. The exact solutions of the KG equation are possible only for certain central potentials such as Hulthen potential [9], Woods-Saxon potential [10], Poschl-Teller potential [11], pseudoharmonic oscillator [12] and reflectionless-type potential [13]. The NU method has been used for solving the hypergeometric type second-order differential equations appeared in the time-independent problems.

In this work, our objective is to solve Klein-Gordon equation for the q-Deformed Morse potential via the NU method.

This paper is organized as follows: In Section 2, we briefly introduce the NU method. We consider one-dimensional KG equation with equal scalar and vector potentials in Section 3. Finally, conclusion is given in Section 4. 


\section{OVERVIEW OF NiKIFOROV-UVAROV METHOD}

This Method [14] has been extensively used to solve the Schrödinger, Dirac, KG and DuffinKemmer-Petiau equations for certain central and non-central potentials. This method in general reduces the second-order linear differential equation to the following form

$$
\frac{\mathrm{d}^{2} \psi(\mathrm{s})}{\mathrm{ds} \mathrm{s}^{2}}+\frac{\bar{\tau}(\mathrm{s})}{\sigma(\mathrm{s})} \frac{\mathrm{d} \psi(\mathrm{s})}{\mathrm{ds}}+\frac{\bar{\sigma}(\mathrm{s})}{\sigma^{2}(\mathrm{~s})} \psi(\mathrm{s})=0
$$

where $\sigma(\mathrm{s})$ and $\bar{\sigma}(\mathrm{s})$ are polynomials, at most second degree, and $\bar{\tau}(\mathrm{s})$ is a polynomial, at most first degree. To find the particular solution of Eq (1), one can use the following form

$$
\psi_{\mathrm{n}}(\mathrm{s})=\phi(\mathrm{s}) \mathrm{y}_{\mathrm{n}}(\mathrm{s})
$$

It reduces to an equation of hypergeometric type

$$
\sigma(s) \frac{d^{2} y_{n}(s)}{d s^{2}}+\tau(s) \frac{d y_{n}(s)}{d s}+\lambda y_{n}(s)=0
$$

where $\lambda$ is a constant given in the form

$\lambda=\lambda_{\mathrm{n}}=-\mathrm{n} \tau^{\prime}(\mathrm{s})-\frac{\mathrm{n}(\mathrm{n}-1)}{2} \sigma^{\prime \prime}(\mathrm{s}), \quad \mathrm{n}=1,2,3, \ldots$

$\phi(s)$ is defined as logarithmic derivative

$\frac{\phi^{\prime}(\mathrm{s})}{\phi(\mathrm{s})}=\frac{\pi(\mathrm{s})}{\sigma(\mathrm{s})}$

$\mathrm{y}_{\mathrm{n}}(\mathrm{s})$ is the hypergeometric-type function whose polynomials solutions are given by Rodrigues relation

$$
y_{n}(s)=\frac{B_{n}}{\rho(s)} \frac{d^{n}}{d s^{n}}\left[\sigma^{n}(s) \rho(s)\right]
$$

where $B_{n}$ is the normalization constant and $\rho(s)$ is the weight function satisfying

$$
\frac{\mathrm{d}}{\mathrm{ds}}[\sigma(\mathrm{s}) \rho(\mathrm{s})]=\tau(\mathrm{s}) \rho(\mathrm{s})
$$

where $\tau(s)=\bar{\tau}(s)+2 \pi(s)$ satisfies the condition $\tau^{\prime}(s)<0$

The function $\pi(\mathrm{s})$ and the parameter $\lambda$, required for this method, are defined as follows: 


$$
\begin{aligned}
& \pi(\mathrm{s})=\frac{\sigma^{\prime}-\bar{\tau}}{2} \pm \sqrt{\left(\frac{\sigma^{\prime}-\bar{\tau}}{2}\right)^{2}-\bar{\sigma}+k \sigma} \\
& \lambda=\mathrm{k}+\pi^{\prime}(\mathrm{s})
\end{aligned}
$$

\section{EIGENFUNCTIONS AND EIGENVALUES}

Let us consider the one-dimensional $\mathrm{KG}$ equation for a spinless particle of rest mass $\mathrm{m}$ and in the natural units $\hbar=\mathrm{c}=1$ :

$$
\frac{\mathrm{d}^{2} \psi(\mathrm{x})}{\mathrm{dx^{2 }}}+\left[(\mathrm{E}-\mathrm{V}(\mathrm{x}))^{2}-(\mathrm{m}+\mathrm{S}(\mathrm{x}))^{2}\right] \psi(\mathrm{x})=0
$$

Where $\mathrm{E}, \mathrm{V}(\mathrm{x})$ and $\mathrm{S}(\mathrm{x})$ are the relativistic energy of the particle, vector and scalar potentials respectively.

Assuming $V(x)=S(x)$, the last equation takes the form

$$
\frac{d^{2} \psi(x)}{d x^{2}}+\left[\left(E^{2}-m^{2}\right)-2(E+m) V(x)\right] \psi(x)=0
$$

Here we consider the general q-Deformed Morse potential as

$$
\mathrm{V}(\mathrm{x})=\mathrm{V}_{0}\left(\mathrm{e}^{-2 \alpha \mathrm{x}}-2 \mathrm{qe} \mathrm{e}^{-\alpha \mathrm{x}}\right)
$$

By substituting $\mathrm{Eq}(12)$ into the relation (11) we have

$$
\frac{d^{2} \psi(x)}{d x^{2}}+\left[\left(E^{2}-m^{2}\right)-2(E+m) V_{0}\left(e^{-2 \alpha x}-2 q e^{-\alpha x}\right)\right] \psi(x)=0
$$

By introducing a new variable $s=v e^{-\alpha x}$ we obtain

$$
\frac{\mathrm{d}^{2} \psi(\mathrm{s})}{\mathrm{ds}}+\frac{1}{\mathrm{~s}} \frac{\mathrm{d} \psi(\mathrm{s})}{\mathrm{ds}}+\frac{1}{\mathrm{~s}^{2}}\left(-\frac{\mathrm{s}^{2}}{4}+\frac{\mathrm{q} v}{2} \mathrm{~s}+\varepsilon_{\mathrm{n}}\right) \psi(\mathrm{s})=0
$$

Where

$$
\varepsilon_{\mathrm{n}}=\frac{\mathrm{E}_{\mathrm{n}}^{2}-\mathrm{m}^{2}}{\alpha^{2}} \text { and } v^{2}=\frac{8(\mathrm{E}+\mathrm{m})}{\alpha^{2}} \mathrm{~V}_{0}
$$

Let us now find the eigenfunctions for this potential. Comparing Eq. (14) with Eq (1), we get 


$$
\bar{\tau}(\mathrm{s})=1, \quad \sigma(\mathrm{s})=\mathrm{s}, \quad \bar{\sigma}(\mathrm{s})=-\frac{\mathrm{s}^{2}}{4}+\frac{\mathrm{qv}}{2} \mathrm{~s}+\varepsilon_{\mathrm{n}}
$$

Substituting the above expressions into relation (8) leads to

$$
\pi(\mathrm{s})= \pm \sqrt{\frac{\mathrm{s}^{2}}{4}+\left(\mathrm{k}-\frac{\mathrm{qv}}{2}\right) \mathrm{s}-\varepsilon_{\mathrm{n}}}
$$

We have the following four possible values of $k$ :

$$
\begin{array}{ll}
\pi(\mathrm{s})= \pm\left(\frac{\mathrm{s}}{2}+\sqrt{-\varepsilon_{\mathrm{n}}}\right) & \text { for } \mathrm{k}_{1}=\frac{\mathrm{q} v}{2}+\sqrt{-\varepsilon_{\mathrm{n}}} \\
\pi(\mathrm{s})= \pm\left(\frac{\mathrm{s}}{2}-\sqrt{-\varepsilon_{\mathrm{n}}}\right) & \text { for } \mathrm{k}_{2}=\frac{\mathrm{q} v}{2}-\sqrt{-\varepsilon_{\mathrm{n}}}
\end{array}
$$

After proper choice of the polynomial $\pi(\mathrm{s})$ and $\mathrm{k}$, we can write the function $\tau(\mathrm{s})$ which has a negative derivative one gets:

$$
\pi(s)=-\left(\frac{s}{2}-\sqrt{-\varepsilon_{n}}\right) \quad \text { for } k=\frac{q v}{2}-\sqrt{-\varepsilon_{n}}
$$

From (5) and (7), we obtain

$$
\begin{aligned}
& \phi(s)=s^{\left(\beta-n-\frac{1}{2}\right)} e^{-\frac{s}{2}} \quad \text { with } \beta=\frac{q v}{2} \\
& \rho(s)=s^{2\left(\beta-n-\frac{1}{2}\right)} e^{-s}
\end{aligned}
$$

Inserting $\mathrm{Eq}(22)$ into Eq. (6), $\mathrm{y}_{\mathrm{n}}(\mathrm{s})$ can be found as follows

$$
y_{n}(s)=B_{n} e^{s} s^{-2 \gamma} \frac{d^{n}}{d s^{n}}\left[e^{-s} s^{2 \gamma+n}\right]
$$

With $\gamma=\beta-\mathrm{n}-\frac{1}{2}$

The eigenfunctions of KG equation take the form

$$
\psi(s)=B_{n} s^{\gamma} e^{-\frac{s}{2}} L_{n}^{2 \gamma}(s)
$$


Where $\mathrm{L}_{\mathrm{n}}^{2 \gamma}(\mathrm{s})$ is the Laguerre polynomials. Using the following formula [16]

$$
\int_{0}^{\infty} \mathrm{s}^{\alpha} \mathrm{e}^{-\mathrm{s}} \mathrm{L}_{\mathrm{n}}^{\alpha}(\mathrm{s}) \mathrm{L}_{\mathrm{m}}^{\alpha}(\mathrm{s}) \mathrm{ds}=\frac{\Gamma(\mathrm{n}+\alpha+1)}{\mathrm{n} !} \delta_{\mathrm{nm}}
$$

The normalization constant. $\mathrm{B}_{\mathrm{n}}$ becomes

$$
B_{n}=\sqrt{\frac{n !}{(n+2 \gamma) !}}
$$

Thus the normalized eigenfunctions can be obtained as

$\psi(s)=\sqrt{\frac{n !}{(n+2 \gamma) !}} s^{\gamma} e^{-\frac{s}{2}} L_{n}^{2 \gamma}(s)$

This result agrees with that of in $\operatorname{Ref}[15]$.

By putting the values of $\tau^{\prime}(\mathrm{s}), \pi^{\prime}(\mathrm{s}), \sigma^{\prime \prime}(\mathrm{s})$ and $\mathrm{k}$ into eqs(4) and (9), the parameter $\lambda$ can be written

$\lambda_{\mathrm{n}}=\mathrm{n}$

$\lambda=\beta-\sqrt{-\varepsilon_{\mathrm{n}}}-\frac{1}{2}$

From eqs(28) and (29) we have

$$
\varepsilon_{\mathrm{n}}=-\left(\beta-\mathrm{n}-\frac{1}{2}\right)^{2}
$$

and using (15) we obtain the energy eigenvalues as

$$
\mathrm{E}_{\mathrm{n}}^{2}-\mathrm{m}^{2}=-\frac{8(\mathrm{E}+\mathrm{m})}{\mathrm{v}^{2}} \mathrm{~V}_{0} \varepsilon_{\mathrm{n}}
$$

This result is the same as in $\operatorname{Ref}[15]$

\section{CONCLUSION}

In this paper, we have obtained the exact solutions of the KG equation for the general deformed Morse potential with equal scalar and vector potentials using the NU method. We have found that the eigenfunctions are obtained in terms of the Laguerre polynomials. Our exact solutions given by eqs (27) and (31) are in exact agreement with the results obtained by solving the KG equation using the algebraic approach [15]. The work concerned with Matlab-graphical user interface for Schrödinger problems is in progress.

\section{ACKNOWLEDGMENT}

The first author expresses his sincere appreciation to his wife for her helpful. 


\section{REFERENCES}

[1] Drazin, P.G. Johnson, R.S. Solitons: An Introduction, Cambridge University Press, Cambridge (1983).

[2] Cooper, F..Khare, A.Sukhatme, U Phys.Rep. 251, 267 (1995)

[3] Batiha, B Australian J. of Basic and Applied Sc. 3(4), 3876-3890 (2009)

[4] Ying Zhang, Phys. Scr. 78, 015006 (2008)

[5] Yasuk, F.Durmus, A Phys. Scr. 77, 015005 ( 2008)

[6] Olgar, E Chin. Phys. Lett. 25(6), 1939 ( 2008)

[7] Soylu, A.Bayrak, O.Boztosun, I J. of Phys. A: Math. and Theor. 41, 065308 ( 2008)

[8] Taskin, F..Boztosun, I.Bayrak, O Int. J. Theor. Phys. 47, 1612-1617 (2008)

[9] Qiang, W.-C.. Zhou R.-S and Gao Y., Phys. Lett. A 371201 (2007)

[10] Guo, J. -Y. Fang, X. -Z. and. Xu, F. -X Phys. Rev. A 66062105 (2002)

[11] Chen G., Acta Phys. Sinica 501651 (2001).

[12] Chen, G.. Chen, Z. D and. Lou, Z. M Chin. Phys. 13279 (2004).

[13] Chen, G. and. Lou, Z.M Acta Phys. Sinica 521071 (2003).

[14] Nikiforov, A. F. and Uvarov, V. B.. Special Function of Mathematical Physics Birkhauser, Bassel, (1988).

[15] Setare, M. R. and Hatami, O. Mod. Phys. Lett A 24361 (2009).

[16] Gradshteyn, I. S. and Ryzhlik, I. M. Tables of Integrals, Series and Products, 5th edn Academic (1994)

\section{Authors}

Said laachir was born in Casablanca - Morocco. He obtained his Ph.D degree in physics in 2006 from the Physics Department of Hassan II University in Morocco. His field of interest includes Electron Paramagnetic resonance (EPR) Spectra Simulation and Informatics. In 2011 he published a book entitled: Simulation of EPR Spectra published by Éditions universitaires européennes- Germany. He has published in

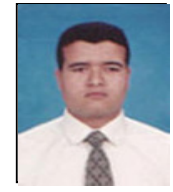
various journals. 